Virginia Commonwealth University VCU Scholars Compass

2005

\title{
Kinetics of ion-induced nucleation in a vapor-gas mixture
}

\author{
Sergey P. Fisenko \\ Virginia Commonwealth University \\ David B. Kane \\ Virginia Commonwealth University \\ M. Samy El-Shall \\ Virginia Commonwealth University, selshall@hsc.vcu.edu
}

Follow this and additional works at: http://scholarscompass.vcu.edu/chem_pubs

Part of the Chemistry Commons

Fisenko, S. P., Kane, D. B., El-Shall, M. S. Kinetics of ion-induced nucleation in a vapor-gas mixture. The Journal of Chemical Physics 123, 104704 (2005). Copyright (C) 2005 AIP Publishing LLC.

\section{Downloaded from}

http://scholarscompass.vcu.edu/chem_pubs/59

This Article is brought to you for free and open access by the Dept. of Chemistry at VCU Scholars Compass. It has been accepted for inclusion in Chemistry Publications by an authorized administrator of VCU Scholars Compass. For more information, please contact libcompass@vcu.edu. 


\title{
Kinetics of ion-induced nucleation in a vapor-gas mixture
}

\author{
Sergey P. Fisenko, ${ }^{\text {a) }}$ David B. Kane,${ }^{\text {b) }}$ and M. Samy El-Shall ${ }^{(\mathbf{c}}$ \\ Department of Chemistry, Virginia Commonwealth University, Richmond, Virginia 23284-2006
}

(Received 23 May 2005; accepted 14 July 2005; published online 13 September 2005)

\begin{abstract}
A general solution for the steady-state ion-induced nucleation kinetics has been derived, considering the differences between ion-induced nucleation and homogeneous nucleation. This solution includes a new effect for nucleation kinetics, the interaction of charged clusters with vapor molecules. Analytical expressions for the ion-induced nucleation rate have been obtained for the limiting cases of high and low thermodynamic barriers. The physical explanation of the so-called sign effect is proposed based on multipole expansion of an electric field of the cluster ion. This theory gives good agreement with experiments and is used to elucidate experimentally observed phenomena. () 2005 American Institute of Physics. [DOI: 10.1063/1.2018632]
\end{abstract}

\section{INTRODUCTION}

The formation of a critical cluster that can act as a nucleus for the spontaneous growth of the new phase is the first step in any first-order phase transition. Nucleation, the mechanism by which the nucleus is formed, is a kinetic process analogous to a chemical reaction that proceeds through a barrier. The nucleation process can be described by the clustering of atoms or molecules from the old phase (vapor) and the loss of molecules from the newly formed clusters back to the vapor, until the clusters pass through a transition state, the nucleus, after which growth of the new phase occurs spontaneously. The process, as with chemical reactions, is driven by the difference in free energy of the two phases. However, in nucleation the formation of the new phase involves overcoming a free-energy barrier, with the nucleus serving as the transition state at the top of the barrier. Thus the formation of the nucleus is the rate limiting step of the phase-transition process. ${ }^{1-3}$

Many species, such as foreign surfaces, long-lived excited-state molecules, ions, and nanoparticles, may serve to lower the thermodynamic barrier to nucleation and thereby increase the nucleation rate, the rate at which nuclei are formed. In particular, ions, because of the strong iondipole and ion-induced dipole interactions, are well known to decrease the thermodynamic barrier to the formation of the nucleus and increase the nucleation rate. Ion-induced nucleation is a phenomenon of great importance for many scientific, environmental, and industrial applications. Among these areas are upper atmospheric chemistry, ${ }^{4-7}$ the formation of jet contrails, ${ }^{8,9}$ and plasma- and chemical-vapor deposition processes. ${ }^{10-12}$

The history of ion-induced nucleation research started approximately 100 years ago with Wilson's original experiments. ${ }^{13}$ While research since that time has greatly in-

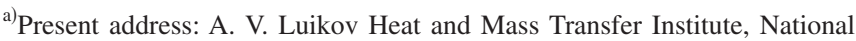
Academy of Sciences of Belarus, Minsk, Belarus.

${ }^{b)}$ Present address: Philip Morris USA Research Center, 4201 Commerce Rd., Richmond, VA 23224.

${ }^{c}$ Author to whom correspondence should be addressed. Electronic mail: selshall@hsc.vcu.edu
}

creased our understanding of the phenomenon, there are still many unsolved problems. In particular, the kinetics of ioninduced nucleation has been practically neglected by researchers. By analogy with chemical reaction kinetics, the expression

$$
J=K \exp \left[-\frac{\Delta \Phi^{*}}{k_{B} T}\right]
$$

is generally used for the nucleation rate $J$. Here $k_{B}$ is the Boltzmann constant, $T$ is the temperature of the vapor-gas mixture, $\Delta \Phi^{*}$ is the free energy required to overcome the thermodynamic barrier, and $K$ is a kinetic prefactor. A second unresolved question is the so called "ion-sign effect," which is an apparent preference for nucleation on ions of either positive or negative charge. While this has been the subject of a number of experimental and theoretical investigations the physical cause of the ion-sign effect remains unresolved. ${ }^{14-17}$ This is in part because of the difficulties involved with the correct analysis of data from ion-induced nucleation experiments, where the inter-related effects of cluster growth, motion, and charging kinetics must all be considered. ${ }^{18-21}$

This paper presents a theoretical treatment of ioninduced nucleation in a mixture of vapor and carrier gas. An explicit formula for the kinetic prefactor $K$ in the nucleation rate equation is developed and the rate equation is used to understand experimental measurements of ion nucleation rates and the electric-field effects observed in ion nucleation experiments. The paper is organized as follows. First we discuss the free energy of formation of charged clusters (Section II). Using a generalized version of the ion nucleation free energy of formation a thermodynamic cause for the ion-sign effect is demonstrated. Next, the kinetics of ion-induced nucleation is considered, and explicit formulas for the nucleation rates are derived for the limiting cases of high and low thermodynamic barriers (Section III). A derivation of the normalization constant for the equilibrium cluster distribution function is presented in the Appendix. In Sec. IV, experimental results obtained in a diffusion cloud chamber are analyzed based on the kinetic theory developed here. The 
kinetic results provide insight to the experimental observations. Preliminary results of this research have been published. $^{22}$

\section{FREE ENERGY OF FORMATION FOR ION NUCLEATION}

A general expression for the free energy of formation of a cluster ion, $\Phi(g)$, is given by

$$
\Phi(g)=a_{1} R^{3}+a_{2} R^{2}+\frac{\varepsilon_{0}}{2}\left[\int_{v} E D d v-\int_{v} E^{2} d v\right],
$$

where $g$ is the number of molecules in the cluster, $R$ is the cluster radius, $E$ is the strength of the electric field, $D$ is the induction field in the cluster, $v$ is the cluster volume, and $\varepsilon_{0}$ is the permittivity of free space. The first two terms in this equation represent the bulk and surface contributions to the free energy, respectively. These two terms together are the classical free energy of cluster formation for homogeneous nucleation. ${ }^{1-3}$ The coefficients $a_{1}$ and $a_{2}$ are given by the classical nucleation theory as ${ }^{3}$

$$
\begin{aligned}
& a_{1}=-\frac{4 \pi k T \ln S}{3 v_{a}}, \\
& a_{2}=4 \pi \sigma,
\end{aligned}
$$

where $S$ is the vapor supersaturation, and $\sigma$ is the surface tension. The last term in Eq. (1) describes the change of free energy due to the electrostatic field. ${ }^{23,24}$

The electrostatic contribution to the free energy of formation is due to the attractive force the ion exerts on the molecules in the cluster. This force significantly reduces the free energy of formation for charged clusters compared with uncharged clusters of the same size. The general description of an ion is a nonuniformly charged and nonspherical object centered in a sphere of radius $r_{i}$. Outside this sphere the electrical field $E(r)$ can be represented by means of a multipole expansion, ${ }^{23,24}$

$$
E(r)=c \frac{q}{r^{2}}+c_{1} \frac{d}{r^{3}} \cdots,
$$

where $c$ and $c_{1}$ are constants, $q$ is the ion charge, $d$ is the ion dipole moment, and the quadrupole and higher moment terms have not been written for simplicity. For nonspherical asymmetric ions it is expected that the dipole and higher moments are not equal to zero.

Using the multipole expansion the electrostatic contribution to the free energy in Eq. (1) is given by

$$
\frac{\varepsilon_{0}}{2}\left[\int_{v} E D d v-\int_{v} E^{2} d v\right]=c^{2} \frac{q^{2}}{r}+2 c c_{1} \frac{q d}{r^{2}}+\cdots .
$$

It follows from Eq. (5) that the contribution of an electrostatic field to the free energy of cluster formation is not fully characterized by the ion charge and radius as in the classic spherical ion approximation made by Thomson. Neglecting the rapidly decreasing higher-order terms it can be shown that the relative contribution of the dipole moment term to the electrostatic free energy is proportional to

$$
\frac{d}{2 q}\left(\frac{1}{R}+\frac{1}{r_{i}}\right)
$$

where $r_{i}$ is the ion radius. For positively charged toluene ions in supersaturated methanol vapor $(S=1.7)$, assuming the dipole moment of the toluene ion is $0.3 \mathrm{D}$ (the dipole moment of the neutral toluene molecule), the relative contribution of the dipole moment to the electrostatic free energy is $\sim 1 \%$ of the total electrostatic free energy, when $R$ is large relative to $r_{i}$. Note that this approximation predicts a preference for nucleation on positive ions, since positive $q$ will decrease the free energy of formation and negative $q$ will increase the free energy of formation.

An ion-sign effect on the nucleation rate has been observed in a number of experimental reports. ${ }^{16}$ However, these experiments are not consistent as to the existence or nature of the sign effect. Equation (6) may help to elucidate these conflicting reports. It predicts that the larger the dipole moment of the ion the larger the ion-sign effect. Therefore, experiments employing different ionization methods that produce different ionic species may be expected to produce different sign effects. Equation (6) also predicts a larger relative contribution from the dipole term for clusters with smaller radii. Since higher supersaturations decrease the size of the critical cluster, it follows that the higher the supersaturation of the vapor the larger the ion-sign effect.

A different approach to describe the ion-dipole interaction was developed by Kusaka et al. ${ }^{14,15}$ The dipole moment considered in their work is the dipole moment of the molecules in the cluster. In a bulk properties approach to the free energy, such as taken here, all contributions of the electrostatic interactions of molecules within the cluster are summarized in the value of the relative dielectric permeability of the condensed phase.

For simplicity we now return to the Thomson model for the free energy of formation. In this approximation, it is assumed that the ion is a uniformly charged sphere located at the center of the cluster. ${ }^{25}$ In this case the dipole and higherorder terms in Eq. (5) vanish and Eq. (1) can be rewritten as

$$
\Phi(g)=a_{1} R^{3}+a_{2} R^{2}+a_{3}\left[\frac{1}{R}-\frac{1}{r_{i}}\right],
$$

with

$$
a_{3}=\frac{q^{2}}{8 \pi \varepsilon_{0}}(1-1 / \varepsilon)
$$

where $\varepsilon$ is the relative dielectric constant of the condensing liquid. In this approximation only the square of the ion charge and the ion radius define the electrostatic contribution to the free energy.

As shown in Fig. 1, the free energy of cluster ion formation, $\Phi(g)$, exhibits two local extrema. The supersaturation significantly affects the shape and position of these extrema. Cluster sizes that correspond to these extrema can be found from the two positive roots of the equation 


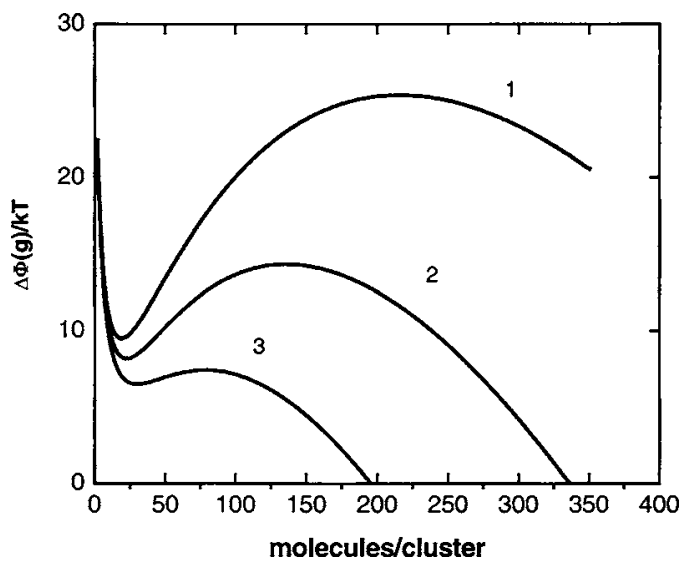

FIG. 1. Free energy of cluster formation, $\Phi(g)$, vs number molecules per cluster at $275 \mathrm{~K}$. Curve 1 is for methanol supersaturation $S=1.67$ (high thermodynamic barrier), curve 2 is for $S=1.78$ (intermediate barrier), and curve 3 is for $S=1.9$ (low barrier). Calculations are for a singly charged ion with a radius of $4.78 \times 10^{-10} \mathrm{~m}$ (the estimation of ion radius for the toluene ion)

$$
\frac{\partial \Phi(g)}{\partial g}=0 .
$$

The larger of the roots gives the critical cluster size for the ion-induced nucleation, $g_{c}$. While an exact analytical solution for $g_{c}$ is too cumbersome, an approximate formula (in terms of the cluster radius) is given by

$$
R_{c} \cong R_{0}+\frac{a_{3}}{3 R_{0}^{3} a_{1}}=R_{0}\left[1-\left(\frac{R_{1}}{R_{0}}\right)^{3}\right],
$$

where $R_{0}$ is the critical cluster size for homogeneous nucleation as calculated from the classical nucleation theory, ${ }^{3}$

$$
R_{0}=\frac{2 \sigma v_{a}}{k T \ln S}=-\frac{2 a_{2}}{3 a_{1}}
$$

and $R_{1}$ is the Raleigh radius as defined by Gamero-Castano and de la Mora, ${ }^{26}$

$$
R_{1}=\sqrt[3]{\frac{a_{3}}{2 a_{2}}}=\sqrt[3]{\frac{(1-1 / \varepsilon) q^{2}}{64 \pi^{2} \varepsilon_{0} \sigma}} .
$$

Note that $R_{1}<R_{0}$ and that the critical cluster size for ioninduced nucleation will always be smaller than the critical cluster size for homogeneous nucleation at the same supersaturation, since $a_{1}<0$ for a supersaturated vapor.

The smaller positive root of Eq. (9) defines the size of the solvated ion cluster, $g_{s}$. An approximate expression for solvated ion cluster radius $R_{s}$ can be written as

$$
R_{s} \cong R_{1}+\frac{a_{1} R_{1}^{2}}{2\left(a_{2}-2 a_{1} R_{1}\right)}=R_{1}\left[1-\frac{1}{4+3 R_{0} / R_{1}}\right] .
$$

The size of the solvated ion cluster is essentially determined by the Raleigh radius $R_{1}$. In a supersaturated vapor the solvated ion clusters form spontaneously and serve as an intermediate species to the nucleus. When nucleation occurs in systems with spatial variations in temperature and supersaturation, such as in a diffusion cloud chamber, the solvated ion clusters and their mobility control many aspects of ioninduced nucleation experiments. ${ }^{19,20}$
For low-vapor supersaturation the two characteristic sizes $R_{s}$ and $R_{c}$ differ greatly (see Fig. 1) and the difference in their free energy of formation, $\Delta \Phi^{*}$, is the thermodynamic barrier for ion-induced nucleation:

$$
\Delta \Phi^{*}=\Phi\left(g_{c}\right)-\Phi\left(g_{s}\right) .
$$

Note that in the Thomson model, the height of the thermodynamic barrier to nucleation is independent of the ion radius.

\section{KINETICS OF ION-INDUCED NUCLEATION}

Nucleation kinetics is the Brownian motion of a cluster over a thermodynamic barrier in cluster size space. ${ }^{27,28}$ The height and shape of the thermodynamic barrier have large effects on the nucleation kinetics. Ion-induced nucleation kinetics differ from homogeneous nucleation kinetics because of the existence of the local free-energy minima corresponding to the solvated ion cluster as well as the maxima corresponding to the nucleus, as shown in Fig. 1.

To describe ion-induced nucleation kinetics we introduce the cluster distribution function $f(g, q, t)$ with three independent variables: the number of molecules in the cluster $g$, charge of the cluster $q$, and the time $t$. For convenience $g$, the number of molecules in the cluster, is used as the independent variable, rather than the cluster radius $R$. If the cluster is assumed to be spherical then the simplest relationship between the cluster radius $R$ and the number of molecules in the cluster $g$ is

$$
\frac{4 \pi}{3} R^{3}=g v_{a}+v_{i}
$$

where $v_{a}$ is the volume per one molecule in the macroscopic liquid phase and $v_{i}$ is the ion volume.

In the following derivation it is assumed that the charge of the clusters remains constant during the nucleation process. Thus the number of independent variables in the cluster distribution function can be reduced to $2, f(g, t)$. For many physical systems ion creation and nucleation are separated in time and space and this simplified description provides a valid picture of the ion-induced induced nucleation process.

In the isothermal approximation, the cluster temperature equals the vapor temperature. Then treating $g$ as a continuous variable, the kinetic equation for the cluster distribution function $f(g, t)$ has the same mathematical form as for homogeneous nucleation. ${ }^{28,29}$ The explicit form of the kinetic equation is

$$
\partial_{t} f(g, t)=\partial_{g}\left\lfloor f(g, t) L_{11} \partial_{g}(\ln f(g, t)+\Phi(g) \beta)\right\rfloor,
$$

where $b=1 / k \mathrm{~T}$ and the coefficient $L_{11}$ is equal to the flux of vapor molecules to the critical cluster,

$$
L_{11}=n k T \Sigma(q) / \sqrt{2 \pi m k T},
$$

with $n$ the vapor density, $\Sigma(q)$ the collisional area of the cluster with the charge $q$, and $m$ the mass of a vapor molecule.

From a theoretical point of view the nucleation rate $J$ is defined by 


$$
\partial_{g}\left[f(g, t) L_{11} \partial_{g}(\ln f(g, t)+\Phi(g) \beta)\right]=-\partial_{g}[J] .
$$

Similar to the homogeneous nucleation rate, the steady-state ion-induced nucleation rate is independent of the variable $g$.

\section{A. Cluster ion-molecule collision cross section}

The collision cross section of a charged cluster with vapor molecules appears in the kinetic coefficient $L_{11}$ in Eq. (17). The role of the attraction between clusters and vapor molecules on homogeneous nucleation kinetics has been studied by Novikov et al., ${ }^{30}$ and by Vasilev and Reiss. ${ }^{31,32}$ However, for ion nucleation long-range Coulomb interactions between a cluster ion and permanent and induced dipole moments of the vapor molecules can significantly increase the collision cross section of the charged cluster compared to a neutral cluster with the same number of molecules. In particular, the collision cross section may be much larger than the geometric size of the cluster. How significant this effect is depends on the cluster size and the magnitude of its permanent dipole moment, ${ }^{33}$ as well as the polarizability and the mean thermal velocity of vapor molecules.

Equation (17) is derived from the kinetic theory of gases $^{34}$ for the free molecular regime. The collision cross section, $\Sigma(q)$, is the cross-sectional area of the sphere with the radius equal to the collision radius of the molecule, $r_{\mathrm{co}}$. The collision radius is defined as the distance at which the potential energy of a molecule in the electrostatic field of the cluster ion is equal to the average kinetic energy of the molecules in the vapor, $(3 / 2) k T$. For a vapor molecule without a permanent dipole moment,

$$
r_{\mathrm{co}}=\left(\frac{\chi q^{2}}{24 \pi^{2} \varepsilon_{0} k T}\right)^{1 / 4}
$$

For a diatomic molecule with a polarazibility of $\chi$ $\cong 13\left(a_{0}\right)^{3}$, where $a_{0}$ is the Bohr radius (the polarazibility of carbon monoxide ${ }^{33}$ ), interacting with a singly charged cluster ion, $r_{\text {co }}$ is equal to $3.7 \times 10^{-10} \mathrm{~m}$. Since the radius of the nucleus, $R_{0}$, is on the order of $10^{-9} \mathrm{~m}$, it follows that the induced dipole moments in the vapor molecules do not significantly affect the collision cross section. However, when the cluster has multiple charges, as may occur in dusty plasmas or astrophysical systems, the increased collision cross section can be substantial.

When the vapor molecule has a permanent dipole moment $d_{0}$, the formula for $r_{\text {co }}$ can be written as

$$
r_{\mathrm{co}}=\left(\frac{q d_{0}}{6 \pi \varepsilon_{0} k T}\right)^{0.5} .
$$

For a singly charged cluster interacting with vapor molecules with a permanent dipole moment $r_{\mathrm{co}}$ is on the order of the size of the nucleus. For example, for methanol molecules at $275 \mathrm{~K}, r_{\mathrm{co}}=1.6 \times 10^{-9} \mathrm{~m}$. This is substantially larger than the critical cluster size for methanol at $S=1.81$. Increasing the charge of the cluster will further increase the value of $r_{\mathrm{co}}$. It also follows from Eq. (20) that the lower the temperature the larger the collision radius for the charged cluster. This behavior coincides with the experimental observations of $\mathrm{He}$ and Hopke. $^{35}$

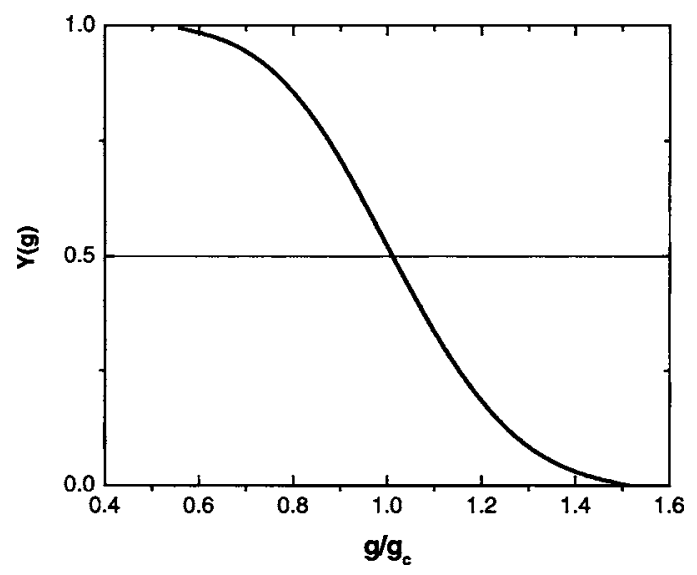

FIG. 2. Universal dependence of a slowly varying function, $y(g)$, vs the normalized number of molecules per cluster, $g / g_{c}$.

\section{B. Solution of the steady-state kinetics equation}

At large cluster sizes we assume the typical Stilzard boundary condition

$$
f(g)=0, \quad g \rightarrow \infty
$$

that effectively removes large clusters from the system. At small cluster sizes the boundary condition is

$$
f\left(g_{s}\right)=f_{e}\left(g_{s}\right),
$$

where the equilibrium cluster ion distribution function is given by

$$
f_{e}(g)=C \exp (-\beta \Phi(g, q)),
$$

where $C$ is a normalization constant. The nonequilibrium distribution function $f(g)$ can then be written as

$$
f(g)=C \exp (-\beta \Phi(g)) y(g),
$$

where the unknown function $y(g)$ describes the deviation of the distribution function $f(g)$ from the equilibrium distribution function $f_{e}(g)$. The function $y(g)$ varies over the interval $0-1$. Its dependence on the reduced cluster size $g / g_{c}$ is displayed in Fig. 2. This function is universal and does not depend on any nucleation parameter.

For these boundary conditions, the steady-state solution of the kinetic equation (16) gives the following expression for the nucleation rate $J:{ }^{29}$

$$
J=\frac{C L_{11}}{\int_{g_{s}}^{\infty} \exp (\beta \Phi(g)) d g} .
$$

The number density of ions, $N_{i}$, is related to the number density of the solvated ion clusters by the normalization of the distribution function for charged clusters,

$$
\int_{0}^{g_{s}} f_{e}(g) d g=N_{i} .
$$

A more detailed derivation of the normalization constant is presented in the Appendix. Using condition (26) the nucleation rate can be rewritten as 


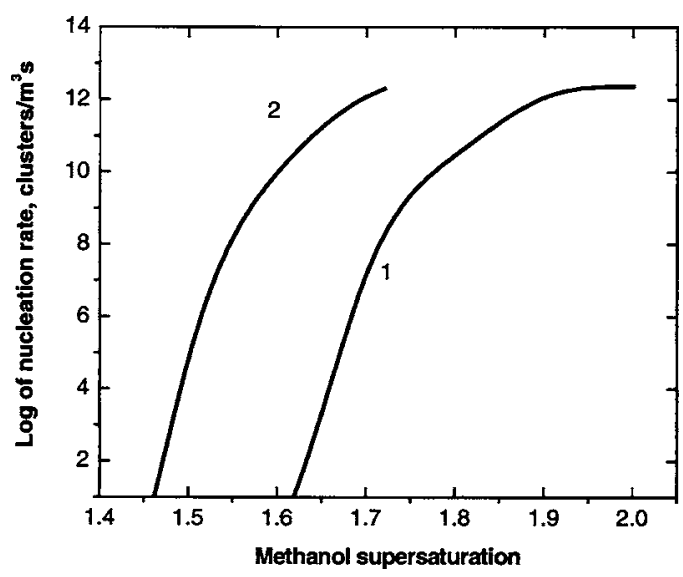

FIG. 3. Ion-induced nucleation rate vs methanol supersaturation at two temperatures: curve 1 for $T=275 \mathrm{~K}$ and curve 2 for $T=295 \mathrm{~K}$. Calculations are for a singly charged ion with a radius of $4.78 \times 10^{-10} \mathrm{~m}$ (the estimation of ion radius for toluene ion), with a total ion density of $10^{6} \mathrm{ions} / \mathrm{m}^{3}$.

$$
J=N_{i} \frac{L_{11}}{\int_{g_{s}}^{\infty} \exp (\beta(\Phi(g))) d g \int_{0}^{g_{s}} \exp (-\beta(\Phi(g))) d g} .
$$

From Eq. (27) it is evident that the nucleation rate is directly proportional to the ion and vapor densities. In classical nucleation theory the homogeneous nucleation rate is directly proportional to the square of the vapor density. Equation (27) is a general expression for the steady-state ioninduced nucleation rate.

Using a numerical solution to Eq. (27) the dependence of the nucleation rate on the vapor supersaturation can be calculated. This dependence is shown at two different temperatures in Fig. 3. The superaturation dependence of the ion nucleation rate is obviously different from the dependence of the homogeneous nucleation rate on supersaturation. For homogeneous nucleation the $\log ($ rate $)$ versus supersaturation curve is nearly linear and has only a slight curvature. However, for ion nucleation the curvature is significant at higher supersaturation. A strong exponential dependence of the nucleation rate on supersaturation is obvious for lower supersaturations. At higher supersaturations the dependence of the nucleation rate on supersaturation is weaker. At low supersaturation the dependence of the ion nucleation rate is similar to that for homogeneous nucleation. But at high supersaturation the kinetics becomes limited by the ion concentration as nearly all ions go on to form nuclei. The temperature dependence of the ion-induced nucleation rate shown in Fig. 3 is primarily due to the decrease in the interfacial surface tension between the cluster and its surroundings as the temperature increases.

The transition from an exponential dependence on superaturation to a weaker dependence suggests that two kinetic domains exist depending on the height of the thermodynamic barrier to nucleation. At low supersaturations there is a high barrier to ion nucleation and, similar to homogeneous nucleation, the kinetics focus on clusters growing over the thermodynamic barrier. At high supersaturations the thermodynamic barrier is low and the formation of the solvated ion cluster dominates the nucleation kinetics. For methanol at
$275 \mathrm{~K}$ the transition between high and low barrier kinetics takes place at supersaturations between 1.7 and 1.8. When the processes near the free-energy minimum and maximum can be separated, as in the extremes of high and low barriers, analytical expressions for the ion-induced nucleation rate can be found.

\section{Steady-state ion-induced nucleation kinetics (high barrier)}

For the high thermodynamic barrier case $\left(\Delta \Phi^{*} / k T \gg 1\right)$, which occurs at low vapor supersaturations and small ion charges, it is possible to separate the kinetics near the solvated ion cluster and the nucleus and focus on clusters growing larger than the critical size. In this case the integrals in Eq. (27) can be approximated in the standard way, ${ }^{29}$ giving

$$
J=\frac{1}{\pi} N_{i} \sqrt{|\alpha| \gamma} L_{11} \exp \left(-\beta \Delta \Phi^{*}\right),
$$

where

$$
\gamma=\frac{1}{2} \partial_{g g}^{2} \Phi\left(g_{s}, q\right) \beta,
$$

and

$$
\alpha=\frac{1}{2} \partial_{g g}^{2} \Phi\left(g_{c}, q\right) \beta
$$

Here $\gamma$ and $\alpha$ are the characteristic curvatures of the free energy of formation at the solvated ion cluster and the critical cluster, respectively. At $S=1.81$ and $T=275 \mathrm{~K}, \alpha=-5$ $\times 10^{-5}$ and $\gamma=6 \times 10^{-3}$. The parameters $\gamma$ and $\alpha$ depend on the charge of the ion, the vapor supersaturation, and the thermophysical properties of the vapor molecules. The smaller the $\gamma$, the broader the local free-energy minimum. When the free-energy minimum is broad, the probability of large fluctuations is small, yielding a lower nucleation rate.

Near the maximum of the thermodynamic barrier, the kinetic equation (16) converts into the diffusion equation. In this region, a well-developed technique ${ }^{36}$ can be used to estimate the characteristic time $\tau_{i}$ for reaching the steady-state regime,

$$
\tau_{i} \sim \frac{(\Delta g)^{2}}{L_{11}}
$$

where $\Delta g$, the characteristic width of thermodynamic barrier expressed as the number of molecules, can be estimated from

$$
\Delta g=|\alpha|^{-1 / 2} .
$$

It follows from expressions (31) and (32) that the broader the thermodynamic barrier, the larger the characteristic time $\tau_{i}$. For ion-induced nucleation numerical estimates show that $\tau_{i}$ is about $10^{-6} \mathrm{~s}$. When the characteristic time over which the macroscopic parameters (temperature, vapor density, and ion concentration) vary is much larger than $\tau_{i}$, it is reasonable to use the steady-state approximation. 


\section{Steady-state ion-induced nucleation kinetics (low barrier)}

High supersaturations or highly charged ions result in a low thermodynamic barrier. When the thermodynamic barrier is on the order of $k_{B} T$, the preceding approximation does not apply and after integration the exact solution of Eq. (25) is

$$
J=N_{i} \frac{2 L_{11}}{\int_{g_{s}}^{\infty_{c}} \exp (\beta(\Phi(g))) d g} \sqrt{\frac{\gamma}{\pi}} \exp \left(\beta \Phi\left(g_{s}\right)\right) .
$$

Estimating the integral in the denominator gives the approximate expression for the ion-induced nucleation rate as

$$
J \cong \frac{2 N_{i}}{\left(g_{c}-g_{s}\right)} \sqrt{\frac{\gamma}{\pi}} L_{11} \frac{\beta\left(\Phi\left(g_{c}\right)-\Phi\left(g_{s}\right)\right)}{\left[\exp \left(\beta\left(\Phi\left(g_{c}\right)-\Phi\left(g_{s}\right)\right)-1\right]\right.} .
$$

From Eq. (31) it is apparent that as the supersaturation increases the nucleation rate also increases due to the decreasing difference between the free-energy minimum and maximum.

\section{Non-steady-state ion-induced nucleation}

To complete our comprehensive analysis of ion-induced nucleation kinetics, we consider the non-steady-state solution of the kinetics equation (16) with the boundary conditions (21) and (22). Non-steady-state nucleation kinetics is important when the system does not have sufficient time to achieve a steady state, such as the initial period after laser ionization. ${ }^{37,38}$ The solution presented here is restricted to the high thermodynamic barrier case and aims to clarify the transition to the steady-state ion-induced nucleation rate.

Let us assume that the initial cluster distribution function has the special form ${ }^{39}$

$$
f(g, 0)=f_{s}(g)+A_{0} \sin \left(\omega\left(g-g_{s}\right)\right),
$$

where $A_{0}$ is defined so that the distribution function is always positive, and $\omega$ is

$$
\omega=\frac{\pi}{g_{u}-g_{s}} .
$$

The upper size limit, $g_{u}$, is defined using expression (32) as

$$
g_{u}=g_{c}+\Delta g .
$$

We seek a distribution function $f(g, t)$ as an approximate solution of Eq. (16) in the form

$$
f(g, t)=f_{s}(g)+A(t) \sin \left(\omega\left(g-g_{s}\right)\right),
$$

where function $A(t)$ will be determined from Eq. (16). Substituting Eq. (38) and applying Galerkin's method, ${ }^{40}$ one obtains an ordinary differential equation for the amplitude $A(t)$,

$$
\frac{d A(t)}{d t}=-A L_{11}\left[\omega^{2}+\omega C_{1}\right],
$$

where the coefficient, $C_{1}$, is defined as

$$
C_{1}=\frac{1}{\left(g_{u}-g_{s}\right)} \int_{g_{s}}^{g_{u}} \sin \left[2 \omega\left(g-g_{s}\right) \partial_{g}(\beta \Phi(g)) d g .\right.
$$

After integrating Eq. (38) we find the solution

$$
A(t)=A_{0} \exp \left[-\omega^{2} L_{11} t\left(1+\frac{C_{1}}{\omega}\right)\right] .
$$

It follows from Eq. (41) that the nucleation rate, $J\left(g_{u}, t\right)$ (the physically observable quantity), can be written as

$$
J\left(g_{u}, t\right)=J+A(t) L_{11} \omega .
$$

From Eq. (42) it is evident that changing the initial cluster ion distribution can control the nucleation rate. Such a mechanism for controlling the nucleation rate could have broad industrial applications.

The characteristic time to establish steady-state nucleation, $\tau$, follows from Eq. (41),

$$
\tau=\frac{1}{\omega^{2} L_{11}\left[1+\frac{C_{1}}{\omega}\right]} \approx \frac{4\left(g_{u}-g_{s}\right)^{2}}{\pi^{2} L_{11}} .
$$

Usually the contribution of the second term in the denominator of expression (43) is significantly smaller than the first term.

If the distribution function $f(g, t)$ is known, then function $A(t)$ can be obtained from

$$
A(t)=\frac{2}{g_{u}-g_{s}} \int_{g_{s}}^{g_{u}}\left[f(g, t)-y(g) f_{e}(g)\right] \sin \left(\omega\left(g-g_{s}\right)\right) d g .
$$

\section{ANALYSIS OF CLOUD CHAMBER EXPERIMENTS}

Ion-induced nucleation takes place in a limited domain of space where the temperature, supersaturation, and ion concentration are such that the nucleation rate is relatively high. This is the case for nucleation experiments performed in a diffusion cloud chamber. ${ }^{19,35,37,38}$ When an external field is applied across the cloud chamber, questions arise about how such a relatively weak electric field influences the nucleation experiments. In this section, the nucleation kinetics model is applied to experimental data generated from ion nucleation experiments utilizing resonance-enhanced multiphoton ionization (REMPI) to create well-characterized molecular ions. ${ }^{19,37,38}$

For the following discussion a brief review of the experimental method will be helpful. More complete details are available in the literature. ${ }^{19-21,37,38}$ These experiments were performed in a diffusion cloud chamber (DCC). ${ }^{41,42}$ The chamber consists of two parallel aluminum plates separated by a glass ring. Both surfaces are covered with a thin film of the working fluid. A steady-state supersaturated vapor is maintained by creating a temperature gradient between the bottom and top surfaces. The gradients of temperature and vapor partial pressure in the chamber are such that the vapor supersaturation reaches a peak at a plane in the chamber that is approximately $\frac{3}{4}$ of the total chamber height. The area 
around the maximum supersaturation profile is referred to as the nucleation zone. Nucleation is detected by light scattering from micron-sized droplets as they fall from the nucleation zone through a $\mathrm{He}-\mathrm{Ne}$ laser beam directed across the bottom half of the chamber.

Ions were generated by REMPI using a coherent pulse of light from an excimer-pumped dye laser at the wavelength of a specific electronic transition of a chromophore molecule. ${ }^{37,38}$ The chromophore is present at a low concentration (mole fractions of $10^{-6}-10^{-4}$ ) in a supersaturated host vapor produced in a DCC. ${ }^{19,37,38}$ In these experiments ions are only produced in the path of the laser beam. For the experiments described here the ionizing laser pulse is directed across the chamber several millimeters below the nucleation zone in the DCC. An electric field between the top and bottom surfaces of the chamber is used to pull the ions to the nucleation zone. ${ }^{19}$ If the maximum nucleation rate in the chamber is sufficiently high, a cloud of droplets produced by ion-induced nucleation is detected within several tenths of seconds after the ionizing laser pulse. Note that no droplets are detected in the absence of a laser pulse or when the wavelength of the light is not tuned to a specific excitation of the chromophore molecule. ${ }^{19,37,38}$ Because of the high density of droplets produced in these experiments single droplet counting is not feasible. Therefore, the intensity of the scattered light-signal is recorded as a function of time by a computer using a photomultiplier tube and an analog-to-digital converter. This "time profile" is then base-line subtracted and integrated to give the total droplet count (TDC), a number proportional to the number of droplets resulting from a laser pulse.

By definition the rate of change of the number of droplets, $N_{d}(t)$, is equal to the nucleation rate,

$$
\frac{d N_{d}(t)}{d t}=J .
$$

For high nucleation rates, as typically observed in these experiments, depletion of the cluster ion density due to nucleation must be taken into account. Using Eq. (27) for the nucleation rate Eq. (45) can be rewritten as

$$
\frac{d N_{d}(t)}{d t}=\left(N_{i}-N_{d}\right) A,
$$

where the reduced form of the nucleation rate, $J=N_{i} A$, is used. After integrating, the total number of droplets is found to be

$$
N_{d}=N_{0}\left(1-\exp \left(-A t^{*}\right)\right)
$$

where $N_{0}$ is the initial number of ions produced by the laser, and $t^{*}$ is the residence time for cluster ions in the nucleation zone,

$$
t^{*} \sim d_{n} / b(E) E
$$

where $d_{n}$ is the length of the nucleation zone, $b(E)$ is the average mobility of charged clusters, and $E$ is the magnitude of the electric field used to direct ions to the nucleation zone. Because cluster growth is an ongoing process, the mobility of the ions depends on their residence time in the supersatu-

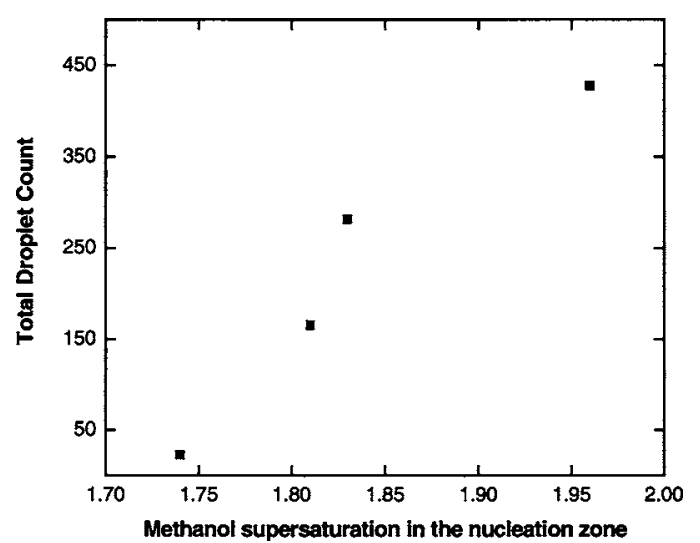

FIG. 4. Experimental total droplet count (TDC) as a function of methanol vapor supersaturation for the nucleation on benzene ions. The TDC is the integrated intensity of the scattered light signal for droplets produced from the average of ten laser pulses $\left(10^{-8} \mathrm{~s}\right)$ tuned to the $6_{0}^{1}$ transition of benzene $\left(38609 \mathrm{~cm}^{-1}\right)$. The temperature of the nucleation zone was $275 \mathrm{~K}$ for all of the measurements.

rated vapor and the degree of supersaturation. ${ }^{19}$

From Eq. (47) it can be shown that, for small nucleation rates, when ion depletion is not significant,

$$
N_{d}=N_{0} A t^{*}
$$

and for high nucleation rates,

$$
N_{d}=N_{0} \text {. }
$$

In the REMPI nucleation experiments the TDC is directly proportional to $N_{d}$. Figure 4 displays the dependence of the total droplet count on the vapor supersaturation for the nucleation of methanol on benzene ions at $275 \mathrm{~K}$. It is evident from this figure that the TDC exhibits the same dependence on supersaturation as the nucleation rate predicted by the kinetics described herein and shown in Fig. 3. At lower supersaturations the TDC rapidly increases, while at higher supersaturations the sharp dependence on TDC is weakened as most ions produce nuclei and the ion concentration decreases rapidly.

From Eqs. (48) and (49) it is evident that for low ion nucleation rates the TDC is dependent on the electric-field strength, while at higher rates it is independent, because all cluster ions serve as nuclei and grow to macroscopic droplets. The experimental data shown in Fig. 5 confirm this conclusion. In these experiments the TDC is measured as a function of the electric-field strength, while the chamber conditions are kept constant. Experiments are presented for two chamber conditions corresponding to low $(S=1.81)$ and high $(S=1.84)$ nucleation rates. At the lower nucleation rate the dependence of the TDC on the electric field is evident, while at the higher nucleation rate no dependence on the electric-field strength is observed.

For relatively low supersaturations corresponding to low nucleation rates the dependence of TDC on the electric-field strength is related to the ion velocity that depends on the electric-field strength ${ }^{43}$ and more weakly on the ion concentration due to recombination kinetics. ${ }^{21}$ At the higher supersaturation, the value of the maximum TDC, displayed in Fig. 5, is approximately seven times larger than that at lower 


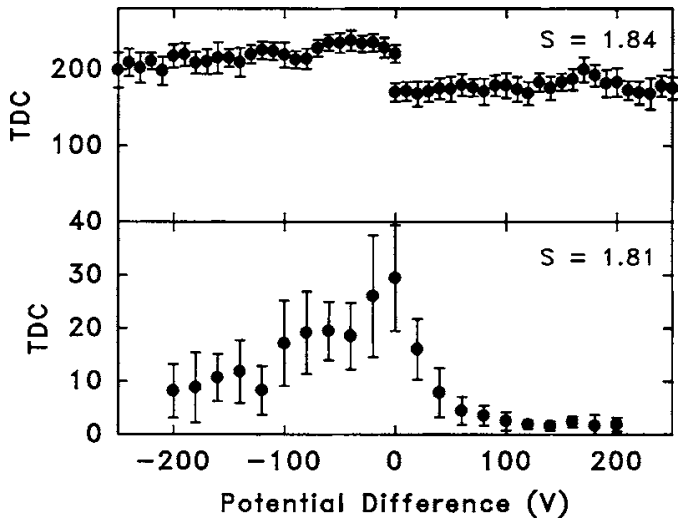

FIG. 5. TDC vs potential difference across the cloud chamber at two methanol vapor supersaturations. The temperature in the nucleation zone is $275 \mathrm{~K}$. Nucleation was induced by REMPI of benzene, the positive ions (negative potentials) are benzene molecular ions $(\mathrm{m} / \mathrm{z}=78)$, and the negative ions (positive potentials) have not been characterized. The height of the cloud chamber is $5 \mathrm{~cm}$. The error bars represent 1 standard deviation of repeated measurements.

supersaturation. Based on the theory developed above we calculate that the nucleation rate is about 3.3 times higher at $S=1.84$ than at $S=1.81$ for $T=275$, in reasonable agreement with the experimentally observed increase in the TDC. Since the TDC is simply the integral of the scattered light signal it is dependent on both the droplet size and concentration. When the supersaturation in the DCC is changed both of these quantities will be affected. From the changes in the droplet growth rate for the two sets of chamber conditions we estimate that changes in droplet size may produce a $10 \%$ change in the TDC. ${ }^{20}$ A supersaturation of 1.795 for the lower supersaturation experiment would be required for a quantitative agreement with the calculated TDC.

An effect due to the polarity of the electric field is obvious in Fig. 5. The primary role of the electric field in these experiments is to direct ions of a particular polarity toward the nucleation zone. For the geometry of these experiments a positive field directs negative ions toward the nucleation zone and a negative field pulls positive ions to the nucleation zone. At $S=1.84$, the TDC for positive ions is approximately $10 \%$ larger than that for negative ions. However, this is not a very convincing proof of the existence of a sign effect in ion nucleation. In these experiments the positively charged benzene ions are produced directly by REMPI. However, the negatively charged ions result from attachment of lowenergy photoelectrons produced by the resonant photoionization of benzene to neutral species. Although the loss of electrons to the chamber walls prior to interacting with neutral molecules in the chamber is expected to be low, the negativeion concentration may be lower than the positive-ion concentration. While the Thomson model does not predict a difference in the nucleation rates on different positive and negative ions (the free-energy barrier is independent of the ion diameter or sign), this difference in nucleation rates is consistent with the multipole description of the electric field used for the free energy of formation for charged clusters.

\section{DISCUSSION AND CONCLUSIONS}

The work reported here is an extensive development of the kinetics of ion-induced nucleation and highlights the dif- ferences between the kinetics of ion nucleation and the much more developed kinetics of homogeneous nucleation. These differences include the existence of a local minimum in the free energy of formation corresponding to the solvated ion cluster, as well as the increased collision cross section of the cluster ion caused by the polarizability and permanent dipole moment of the vapor-phase molecules.

It was shown that Thomson's expression for the free energy of charged cluster formation includes only the first term of the multipole expansion. When the electric field near the cluster ion is not spherically symmetric, the dipole and higher-order moments of the cluster ion charge distribution give an additional contribution to the electrostatic term of the free energy of cluster formation. This contribution may be a physical foundation for the "ion-sign" effect observed in ion nucleation experiments. This multipole expansion model suggests a preference for nucleation on positive ions that is expected to increase as the critical cluster size decreases, i.e., as the supersaturation increases.

As with homogeneous nucleation, the ion nucleation rate exhibits an exponential dependence on the height of the freeenergy barrier to nucleation. However, for ion nucleation this barrier is no longer the difference in free energy between the monomer and the critical cluster, but rather the difference in free energy between the solvated ion cluster (the local freeenergy minimum) and the critical cluster (the free-energy maximum). In the framework of Thomson's model, this barrier height is independent of the ion radius and depends only on the charge squared. The ion nucleation rate is directly proportional to the number densities of solvated ions and the vapor molecules, while for homogeneous nucleation the rate is proportional to the square of the number density of the vapor molecules. When the thermodynamic barrier to nucleation is high the steady-state ion-induced nucleation rate depends on the product of the characteristic curvatures of the extrema of the cluster free energy [Eqs. (29) and (30)]. For relatively high supersaturation when the height of the thermodynamic barrier is on the order of $k_{B} T$, the kinetics of ion-induced nucleation reduces to pure diffusion kinetics.

The rate of ion-induced nucleation is affected by the dipole moment of the host vapor molecules. This dipole moment determines the collision cross section of the solvated ion with vapor molecules. This effect is stronger for low temperatures, and may play an important role in particle formation in systems such as the interstellar medium. This is a large deviation from homogeneous nucleation kinetics, and suggests that an additional parameter, the vapor molecule dipole moment, is necessary for a complete description of experimental results.

The kinetics model has been applied to evaluate data from nucleation experiments in a diffusion cloud chamber. This model explains that the TDC does not continue to increase exponentially as the supersaturation is increased because of the transition to the low barrier kinetics where the rate becomes limited by the ion concentration. In some circumstances multiple nucleation mechanisms may be active simultaneously. Comparing the rates of ion-induced, $J$, and homogeneous nucleation, $I$, at the same supersaturation and temperature it is found that 


$$
\frac{J}{I} \approx \frac{N_{i}}{n} \exp \left[\beta\left(\Delta \Phi_{h}^{*}-\Delta \Phi^{*}\right)\right],
$$

where $\Delta \Phi_{h}^{*}$ is the free energy of the critical cluster for homogeneous nucleation. Since the number density of the ions will generally be orders of magnitude smaller than the number density of the vapor molecules, it may be possible for more droplets (particles) to be created by homogeneous nucleation rather than by ion-induced nucleation. This complicates the analysis of experimental results obtained at high supersaturation as two channels of nucleation may exist.

Experimental measurement of the TDC versus the strength of electric field confirms our theoretical results for steady-state ion-induced nucleation rate. At a lower supersaturation the nucleation rate is dependent on the electricfield strength while the dependence is not observed at the higher supersaturation. This indicates the transition between the kinetics at high thermodynamic barriers where the TDC is dependent on the residence time of the ions in the nucleation zone and at low thermodynamic barriers where the TDC is limited by the ion number density.

Future work will include expanding this model to multicomponent ion-induced nucleation, since this is a subject of significant scientific interest with vast technological applications. The development of binary ion-induced nucleation is currently in progress in our laboratory. The development of a general theory of ion-induced nucleation, which will include fluctuations of the cluster charge during nucleation, is also very desirable.

\section{ACKNOWLEDGMENTS}

The authors gratefully acknowledge support from the National Research Council's Twinning Program for support in the initiation of the collaboration that resulted in this work. Acknowledgement is made to the Donors of the Petroleum Research Fund, administered by the American Chemical Society (PRF No. 37582-AC6), and to NASA (NNG04GH45G) for the partial support of this research.

\section{APPENDIX: ION-INDUCED NUCLEATION}

In this Appendix we present a more detailed derivation of the normalization constant for the equilibrium cluster ion distribution function. The equilibrium distribution function $f_{e}(g, q)$ that defines the number density of different cluster species can be written as

$$
f_{e}(g, q)=C \exp [-\beta \Delta \Phi(g, q)],
$$

where $C$ is the normalization constant. The constant $C$ is defined so that the density of clusters of $g$ molecules around an ion, $n_{g}$, is given by

$$
n_{g}=C \exp [-\beta \Delta \Phi(g, q)] \text {. }
$$

To calculate $C$, a crucial assumption is made, that the total density of charged clusters is approximately equal to the sum of the densities $n_{1}$ and $n_{2}$. In other words, most of the charged clusters are either single molecules or dimers clustered with an ion. Using the rate equations (A3) and (A4), $n_{1}$ and $n_{2}$ can be calculated, ${ }^{30}$

$$
\begin{aligned}
& \frac{d\left\langle n_{1}\right\rangle}{d t}=k_{1}\langle n\rangle\left\langle n_{i}\right\rangle-k_{1}^{*}\left\langle n_{1}\right\rangle, \\
& \frac{d\left\langle n_{2}\right\rangle}{d t}=k_{2}\langle n\rangle\left\langle n_{1}\right\rangle-k_{2}^{*}\left\langle n_{2}\right\rangle .
\end{aligned}
$$

Here $\left\langle n_{x}\right\rangle$ denotes the concentration of species $x$ in the reaction scheme. In particular, $\langle n\rangle$ is the concentration of vapor molecules and $\left\langle n_{i}\right\rangle$ is the concentration of unclustered ions (bare ions). The forward and back reaction rates are denoted by $k_{g}$ and $k_{g}^{*}$, respectively. Other reaction pathways have been neglected because the concentrations of larger clusters are significantly small. At equilibrium,

$$
\frac{k_{1}}{k_{1}^{*}}=\exp (-\beta \Delta \Phi(1, q)),
$$

and

$$
\frac{k_{2}}{k_{2}^{*}}=\exp (-\beta \Delta \Phi(2, q)) .
$$

So from Eqs. (A3) and (A4) we have the following relationships:

$$
\begin{aligned}
& \left\langle n_{1}\right\rangle=\exp (-\beta \Delta \Phi(1, q))\langle n\rangle\left\langle n_{i}\right\rangle, \\
& \left\langle n_{2}\right\rangle=\exp (-\beta \Delta \Phi(1, q)-\beta \Delta \Phi(2, q))\langle n\rangle^{2}\left\langle n_{i}\right\rangle .
\end{aligned}
$$

Changing from concentration back to the number density and using Eq. (A2) it is found that

$$
C=n_{i} \frac{n^{2}}{\left(n_{i}+n\right)^{2}} \exp [-\beta \Delta \Phi(1, q)] .
$$

If the number density of ions is much less than the number density of the vapor molecules, Eq. (A9) gives

$$
C=n_{i} \exp [-\beta \Delta \Phi(1, q)],
$$

and when $\beta \Delta \Phi(1, q) \ll 1$ the normalization coefficient can be approximated by

$$
C \approx n_{i},
$$

as was used in Eq. (26). Note that for homogeneous nucleation (i.e., $n_{i}=0$ ) the above derivation gives $C \approx n$, as in the classical theory for homogeneous nucleation.

Mass spectrometric investigation of the initial ionmolecule clustering steps ${ }^{44}$ shows that for reaction of benzene ions $\left(\mathrm{C}_{6} \mathrm{H}_{6}^{+}\right)$with methanol molecules at room temperature, $\beta \Delta \Phi(1, q) \sim 8 .^{45}$ This suggests that the approximation of the normalization coefficient used in Eq. (26) may lead to a discrepancy between experimental and calculated nucleation rates of up to three orders of magnitude.

\footnotetext{
${ }^{1}$ D. Kashchiev, Nucleation: Basic Theory with Applications (Butterworths-Heinemann, Oxford, 2000).

${ }^{2}$ F. F. Abraham, Homogeneous Nucleation Theory (Academic, New York, 1974).

${ }^{3}$ A. C. Zettlemayer, Nucleation Phenomena (Elsevier, Amsterdam, 1977).

${ }^{4}$ O. I. Shumilov, E. A. Kasatkina, K. Henriksen, and E. V. Vashenyuk, Ann. Geophys. 14, 1119 (1996).

${ }^{5}$ S. H. Lee, J. M. Reeves, J. C. Wilson, D. E. Hunton, A. A. Viggiano, T. M. Miller, J. O. Ballenthin, and L. R. Lait, Science 301, 1886 (2003).

${ }^{6}$ L. Laakso, M. Kulmala, and K. E. J. Lehtinen, J. Geophys. Res., [Atmos.]
} 
108, 4574 (2003).

${ }^{7}$ L. Laakso, J. M. Makela, L. Pirjola, and M. Kulmala, J. Geophys. Res., [Atmos.] 107, 4427 (2002).

${ }^{8}$ F. Q. Yu and R. P. Turco, Geophys. Res. Lett. 25, 313 (1998).

${ }^{9}$ F. Q. Yu, R. P. Turco, and B. Karcher, J. Geophys. Res., [Atmos.] 104, 4079 (1999).

${ }^{10}$ M. Adachi, S. Tsukui, and K. Okuyama, J. Nanopart. Res. 5, 31 (2003).

${ }^{11}$ K. Nakaso, B. Han, K. H. Ahn, M. Choi, and K. Okuyama, J. Aerosol Sci. 34, 869 (2003).

${ }^{12}$ S. Sattel, J. Gerber, and H. Ehrhardt, Phys. Status Solidi A 154, 141 (1996).

${ }^{13}$ C. Wilson, Philos. Trans. R. Soc. London 189, 256 (1897).

${ }^{14}$ I. Kusaka, Z. G. Wang, and J. H. Seinfeld, J. Chem. Phys. 102, 913 (1995).

${ }^{15}$ I. Kusaka, Z. G. Wang, and J. H. Seinfeld, J. Chem. Phys. 103, 8993 (1995).

${ }^{16}$ H. Rabeony and P. Mirabel, J. Phys. Chem. 91, 1815 (1987).

${ }^{17}$ A. I. Rusanov and F. M. Kuni, J. Colloid Interface Sci. 100, 264 (1984).

${ }^{18}$ T. Matsoukas and M. Russell, Phys. Rev. A 55, 991 (1997).

${ }^{19}$ D. Kane, M. Rusyniak, S. P. Fisenko, and M. S. El-Shall, J. Phys. Chem. 104, 4912 (2000)

${ }^{20}$ D. Kane, S. P. Fisenko, and M. S. El-Shall, Chem. Phys. Lett. 277, 13 (1997).

${ }^{21}$ D. Kane, S. Fisenko, and M. S. El-Shall, Chem. Phys. Lett. 277, 6 (1997).

${ }^{22}$ S. P. Fisenko, D. B. Kane, and M. S. El-Shall, in Nucleation and Atmospheric Aerosols, edited by B. Hale and M. Kulmala, AIP Conf. Proc. No. 534 (AIP, New York, 2000), p. 103.

${ }^{23}$ L. D. Landau and E. M. Lifshitz, Electrodynamics of Continuous Media (Pergamon, Oxford, 1984).

${ }^{24}$ J. D. Jackson, Classical Electrodynamics (Wiley, New York, 1962).

${ }^{25}$ J. J. Thomson and G. P. Thomson, Conduction of Electricity Through Gases, 3rd ed. (Cambridge University Press, Cambridge, 1928).

${ }^{26}$ M. Gamero-Castano and J. F. de la Mora, J. Chem. Phys. 117, 3345 (2002).

${ }^{27}$ A. G. Bashkirov and S. P. Fisenko, Theor. Math. Phys. 48, 636 (1981).
${ }^{28}$ A. G. Bashkirov, Theor. Math. Phys. 1, 203 (1970).

${ }^{29}$ H. Risken, The Fokker-Planck Equation (Springer, Berlin, 1989).

${ }^{30}$ V. M. Novikov, O. V. Vasilev, and H. Reiss, Phys. Rev. E 55, 5743 (1997).

${ }^{31}$ O. V. Vasilev and H. Reiss, Phys. Rev. E 54, 3950 (1996).

${ }^{32}$ O. V. Vasilev and H. Reiss, J. Chem. Phys. 105, 2946 (1996).

${ }^{33}$ Y. P. Raizer, Gas Discharge Physics (Springer, Berlin, 1991).

${ }^{34}$ J. H. Ferziger and H. G. Kaper, Mathematical Theory of Transport Processes in Gasses (North-Holland, Amsterdam, 1972).

${ }^{35}$ F. He and P. K. Hopke, J. Chem. Phys. 99, 9972 (1993).

${ }^{36} \mathrm{P}$. Morse and H. Feshbach, Methods of Theoretical Physics (McGrawHill, New York, 1999).

${ }^{37}$ D. B. Kane, G. M. Daly, and M. S. El-Shall, J. Phys. Chem. 99, 7867 (1995).

${ }^{38}$ D. Kane and M. S. El-Shall, Chem. Phys. Lett. 259, 482 (1996).

${ }^{39}$ Following the basic ideas of the Galerkin method, the arbitrary distribution function $f(g, t)$ can be represented as $f(g, t)=f_{s}(g)+\sum_{i}\left[A_{i}(t) \sin (\omega(g\right.$ $\left.-g) i)+B_{i}(t) \cos (\omega(g-g) i)\right]$. Only the functions $A_{i}(t)$ and $B_{i}(t)$ depend on time. The maximum contribution to the relaxation process of the distribution function to the steady state is given by the $i=1$ terms. These terms have maximal "length" in cluster size space. Characteristic relaxation time of the functions $A_{i}$ and $B_{i}$, is the characteristic relaxation time of the distribution function $\tau$. To simplify the calculation of the relaxation time we take only the term with $\sin (\omega(g-g s))$. Therefore, for this special case, we immediately have the initial condition, used in the text [Eq. (35)], $f(g, 0)=f_{s}(g)+A_{0} \sin \left(\omega\left(g-g_{s}\right)\right)$, where $A_{0}$ is initial constant

${ }^{40}$ C. A. Fletcher, Compuational Galerkin Methods (Springer, New York, 1984).

${ }^{41}$ D. Kane and M. S. El-Shall, J. Chem. Phys. 105, 7617 (1996).

${ }^{42}$ M. Rusyniak, V. Abdelsayed, J. Campbell, and M. S. El-Shall, J. Phys. Chem. B 105, 11866 (2001)

${ }^{43}$ T. Seto, K. Okuyama, L. de Juan, and J. Fernandez de la Mora, J. Chem. Phys. 107, 1576 (1997).

${ }^{44}$ G. M. Daly, M. Meot-Ner, Y. B. Pithawalla, and M. S. El-Shall, J. Chem. Phys. 104, 7965 (1996).

${ }^{45}$ M. Mautner and M. S. El-Shall (unpublished). 